\title{
Laguistas et pescadores
}

L'apprentissage de la pêche dans une région côtière de l'Amazonie (Vila Sucuriju, Brésil)

\section{Carlos Emanuel Sautchuk}

\section{(2) OpenEdition}

12 Journals

Édition électronique

URL : https://journals.openedition.org/tc/1517

DOI : $10.4000 /$ tc. 1517

ISSN : 1952-420X

Éditeur

Éditions de l'EHESS

\section{Édition imprimée}

Date de publication : 1 juin 2005

ISSN : 0248-6016

\section{Référence électronique}

Carlos Emanuel Sautchuk, "Laguistas et pescadores », Techniques \& Culture [En ligne], 45 | 2005, mis en ligne le 22 mai 2008, consulté le 29 septembre 2022. URL : http://journals.openedition.org/tc/1517 ; DOI : https://doi.org/10.4000/tc. 1517

Ce document a été généré automatiquement le 29 septembre 2022.

Tous droits réservés 


\title{
Laguistas et pescadores
}

L'apprentissage de la pêche dans une région côtière de l'Amazonie (Vila Sucuriju, Brésil)

\author{
Carlos Emanuel Sautchuk
}

\begin{abstract}
"Transmission de la forme des techniques Dernier point de vue : l'enseignement des techniques étant essentiel, nous pouvons les classer par rapport à la nature de cette éducation et de ce dressage. » (Marcel Mauss, Les techniques du corps, 1934)
\end{abstract}

L'apprentissage de la pêche est abordé ici selon trois perspectives. La première consiste dans le primat de l'action ou du geste dans le cadre de l'analyse de la technique; c'est là un des principaux legs d'André Leroi-Gourhan (1984, 1987 et 1992). On prendra aussi en considération que ce qui est transmis, c'est le travail, entendu comme un ensemble de facteurs particulier et complexe, et non simplement un savoir ou un savoir-faire (Delbos \& Jorion 1984: 46). Enfin, on rappellera que la maitrise d'une technique est aussi l'affirmation d'une identité (Geistdoerfer 1980 : 913; Ingold 2000 : 318). De telles perspectives conduisent à estimer insuffisante la compréhension du procès d'apprentissage en termes d'intériorisation d'un savoir par l'individu (à travers la transmission orale d'un savoir abstrait ou par l'imitation des comportements), qui évolue cumulativement et prend fin à l'âge adulte. L'apprentissage semble mieux défini en tant qu'adaptation de l'individu pour s'articuler à un système de relations techniques et symboliques (entre objets, animaux, humains, etc.). Peut-être est-ce plus évident pendant la période de maturation, mais cela continue à répondre à des transformations ultérieures. Bref, si l'on comprend l'apprentissage, au sens large, comme une série de transformations du travailleur face à une activité, ce procès apparait déterminé par les caractéristiques particulières des systèmes techniques et symboliques, ce qui comprend tant le statut de l'homme même que son rapport avec les autres éléments en jeu. Au travers du contraste entre deux types de pêche pratiqués par les hommes dans un petit village sur la côte de l'Amazonie, le présent article 
cherche à montrer que l'apprentissage d'une habileté technique peut assumer des rôles et connaître des développements différents selon les activités.

2 Vila Sucuriju, qui compte près de 520 habitants, est situé à l'embouchure de la rivière éponyme (le Sucuriju), au Cabo Norte, entre l'estuaire de l'Amazone et la Guyane Française $\left(01^{\circ} 39^{\prime} 49^{\prime \prime} \mathrm{N} / 49^{\circ} 55^{\prime} 43^{\prime \prime} \mathrm{W}\right)$. Bien que côtier, ce village présente des caractéristiques insulaires, car les liaisons avec d'autres communautés et centres urbains ne passent que par la mer (en 12 heures de voyage ou plus). Et cela parce que la région environnante se compose de mangroves, de terres inondées et de lacs qui empêchent que l'on y accède par le continent. Le village comprend 96 maisons, disposées le long d'une sorte de quai d'un peu plus d'un kilomètre, en une ligne droite, qui borde la rivière. Ce quai et toutes les autres constructions sont en bois et sur pilotis, car lors de certains lanços (marées de syzygie), l'eau salée couvre tout le terrain, jusqu'à rejoindre, pendant la saison des pluies, la mangrove qui se trouve derrière le village. En raison de cette arrivée constante des marées dans la rivière, ce sont les eaux de pluie que l'on utilise pour la consommation. Pour la saison sèche (d'août à décembre), les eaux pluviales sont stockées dans deux citernes. Pratiquement tous les sols de la région sont inondés d'eau douce, ou inondables par de l'eau salée, ce qui contribue à expliquer l'absence d'agriculture.

3 L'activité économique est la pêche, pratiquée dans deux environnements : en mer et sur les lacs ${ }^{1}$. Identifiés de façon contrastée et jouant un rôle central dans la cosmologie locale, ces deux milieux impliquent des techniques et des dynamiques de travail différentes. Sur les lacs, les pêcheurs manient des pirogues (montarias) pour deux rameurs (photo 1), usant surtout du harpon pour attraper essentiellement le pirarucu (Arapaïma gigas), le plus grand poisson de l'Amazonie. En mer, ce sont des bateaux motorisés, avec un équipage de trois à six pêcheurs, qui retirent de l'eau avant tout la gurijuba (Arius parkeri) à l'aide de palangres (espinhel), lignes de fond d'à peu près $1,5 \mathrm{~km}$, munies d'environ 500 hameçons et 14 ancres $^{2}$. Les pêcheurs se sont spécialisés dans une des deux techniques, qu'ils se transmettent suivant la parenté. Il y a 49 familles de pêcheurs en mer-pescadores- et 32 familles de pêcheurs de lac-laguistas-marquées par une tendance endogamique.

4 Pour la pêche côtière, la maîtrise de l'« horaire » -à savoir de la cadence des maréesest centrale. Dans cette région de macro-marées (jusqu'à 10 mètres de variation), de forts courants et d'eaux peu profondes, la navigation, la protection du bateau et le bon fonctionnement des engins de pêche dépendent de la stricte synchronie avec l'hydrodynamique. Toutefois, outre le rapport bateau/mer, l'activité des pêcheurs doit se plier aussi aux tâches bien définies du système de travail à bord. De fait, ce qui régit le rythme du travail en mer, c'est le «bateau» (barco) -lato sensu: embarcation, instruments, moteur et équipage (photo 2)-, et cela à deux niveaux.

Unité symbolique, de production et de crédit, c'est chacun des 32 bateaux qui se déplace en mer à la recherche du poisson. Mais tous présentent aussi une dynamique interne de travail à bord; les activités individuelles du pêcheur sont donc coordonnées selon le rythme de l'activité du tout (tirer et «cueillir » le fil, jeter l'ancre, etc.). Il est des signes de prestige du bon pescador: son "effort " (esforço) et sa "disposition " (disposição), aussi bien que le fait d'avoir travaillé sur plusieurs bateaux. Dans le domaine maritime, les explications du succès ou de l'échec de la pêche mettent en rapport des arguments techniques, une éthique du travail et des éléments du catholicisme populaire local. 


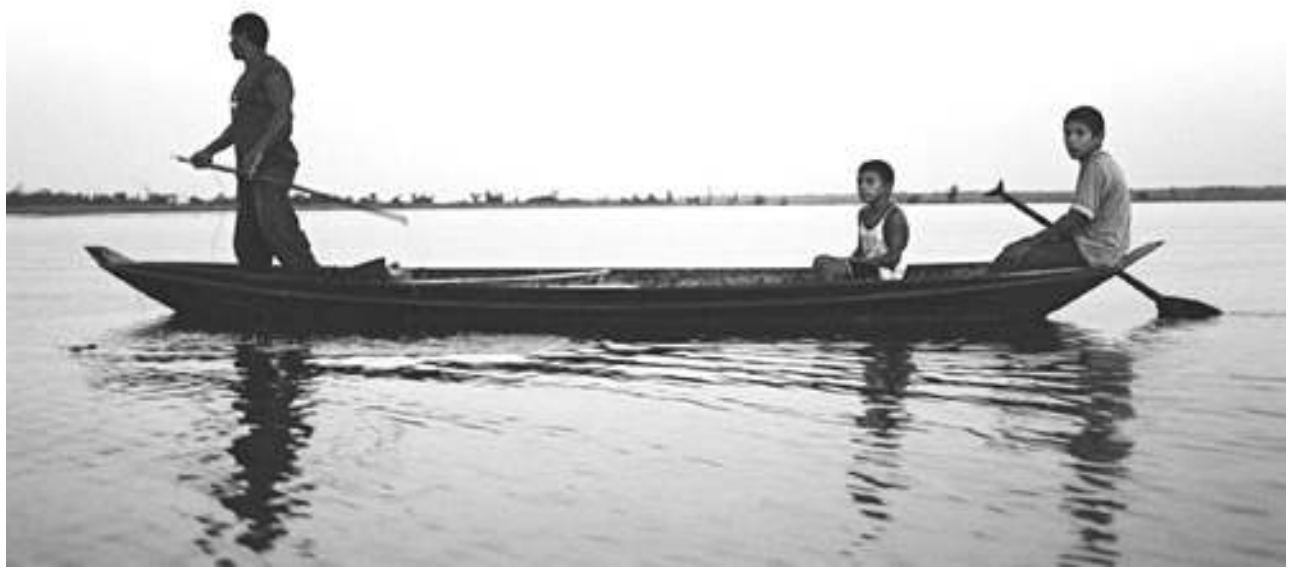

Photo 1. Une pirogue avec le proeiro Antenor et ses deux fils : Bicudo, 12 ans, comme piloto et Passarinho, 7 ans, au centre

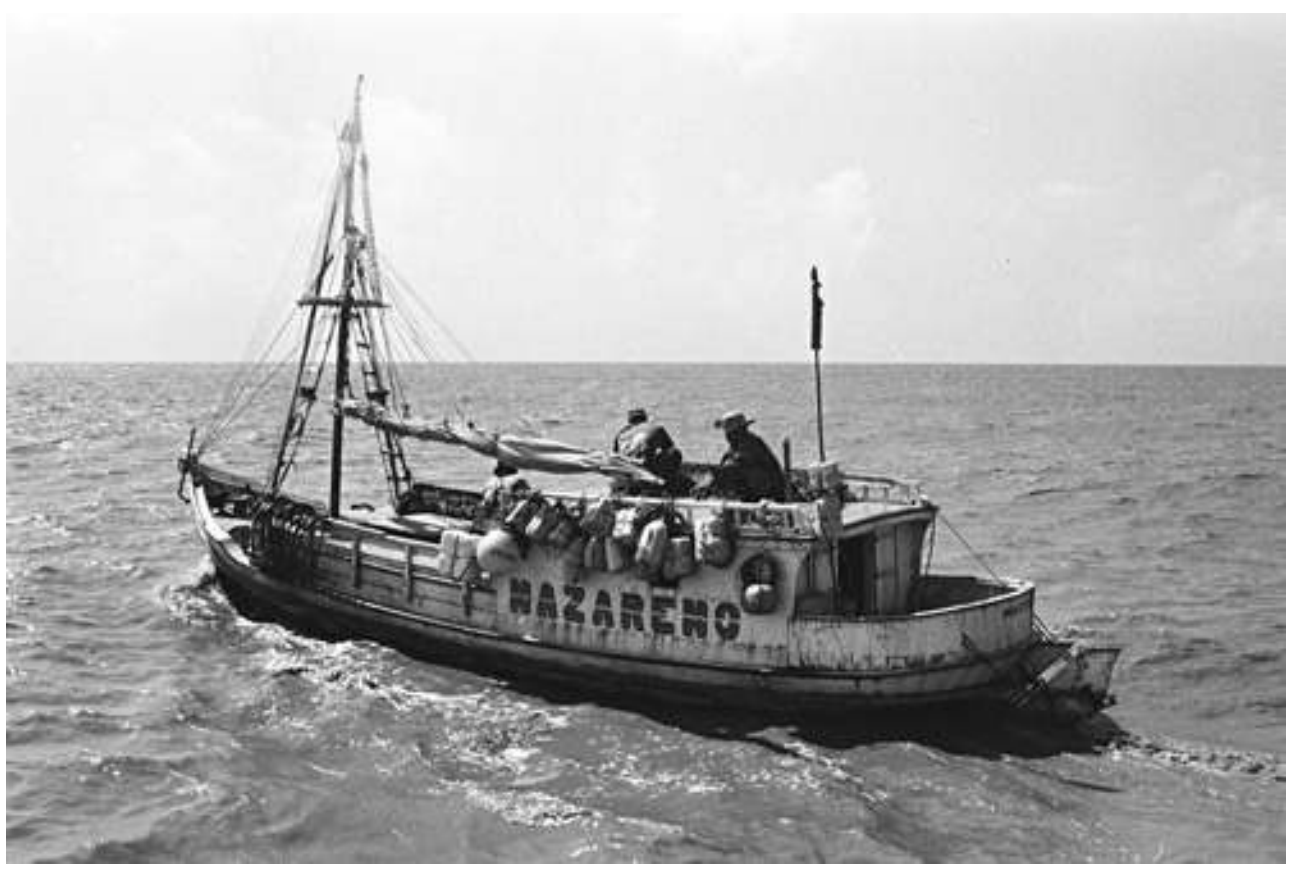

Photo 2. Bateau partant à la pêche. De ce côté, on voit les ancres et les bouées ; les pescadores (Mira, Jeandré et Nei) appâtent les hameçons et rangent la ligne de l'autre côté

6 "Dans» le lac (le mot est toujours utilisé au singulier, mais indique une zone comprenant plusieurs lacs aux noms divers), milieu réputé plus beau et plus agréable, le laguista est en rapport avec d'autres êtres, qui ont des caractéristiques propres et des comportements imprévisibles. On peut certes toujours avoir à affronter l'« astuce » d'un pirarucu, l'«agressivité» d'un crocodile (jacaré-açu, Melanosuchus niger), l'« audace » d'un sucuriju (Eunectes murinus) -anaconda dont un spécimen énorme fut le démiurge de la rivière et du village-, mais aussi des entités surnaturelles, comme les maîtres des animaux dans certaines régions. Plusieurs de ces êtres et animaux sont 
parfois invisibles ou peuvent changer d'apparences. Ils exercent des influences sur le comportement et les aptitudes de l'homme, ce qui peut être la cause d'un harponnage raté, d'une disparition subite des poissons ou de la transformation d'un pêcheur en pirarucu.

Ici, l'entité prééminente des points de vue symbolique, technique et économique, c'est le proeiro, le pêcheur qui se tient à la proue. Ce spécialiste du harponnage parcourt les lacs avec sa pirogue et son piloto (qui est à la poupe et pilote la pirogue). La capture est fonction du rapport d'affrontement et d'astuce du proeiro avec le pirarucu, poisson "fin", « intelligent» et "coquin » (velhaco), apte à tromper l'homme. Voici le signe distinctif du proeiro: sa "pratique», y compris sa maitrise du harpon, le savoir concernant le comportement du poisson, et le fait d'avoir piloté la pirogue pour des pêcheurs prestigieux.

8 Les tâches consistant à lancer l'hameçon à la mer et à harponner le pirarucu sont jugées les actions les plus significatives, respectivement pour les pescadores et pour les laguistas. Mais harpon et hameçon ne sont pas de simples instruments, ni doués de propriétés similaires. Le premier est une extension sensori-motrice du proeiro, il le lie à distance au poisson. Objet personnel, il participe aussi de l'aptitude du proeiro; quand celui-ci veut se faire valoir, il proclame: "Ce harpon-là a déjà tué beaucoup de pirarucu» (photo 3). L'hameçon, par contre, est un partenaire indispensable pour la pêche maritime, mais il lui arrive de "trahir ». C'est à cause de sa forme et de son mouvement (qui résulte de l'interaction entre le bateau et le courant) qu'il peut capturer tout seul le poisson, mais aussi le pêcheur lui-même. Ce danger est évoqué par des dictons comme celui-là : «L'hameçon n'a qu'une pointe, mais il ne rate pas ».

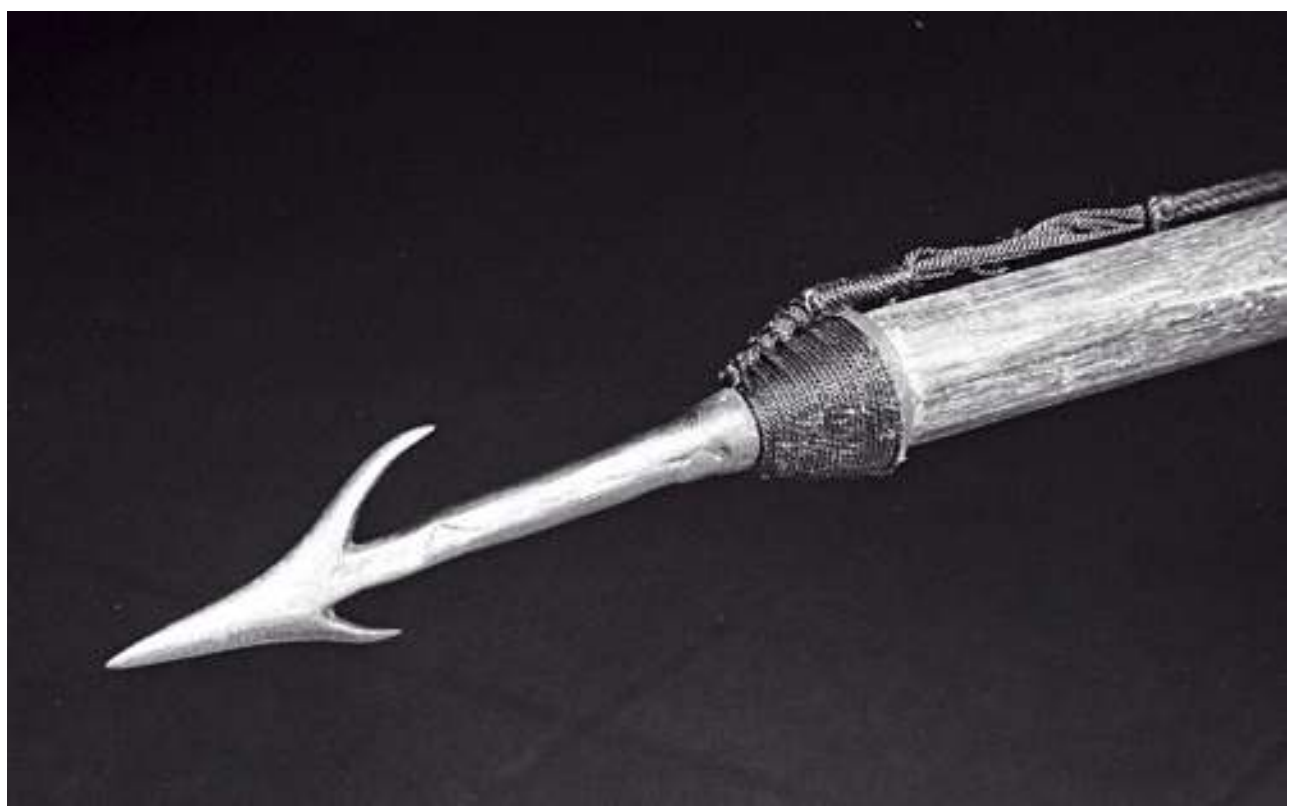

Photo 3. La pointe du harpon est en métal et mesure de 15 à $25 \mathrm{~cm}$. Elle se détache de la hampe qui est en bois plus dense que l'eau et mesure entre 2 et 2,8 $\mathrm{m}$ de long

Apparus dès la création de Vila Sucuriju -il y a environ un siècle-, les termes pescador et laguista désignent des personnes issues des îles de l'estuaire de l'Amazone et habitant un même lieu, qui travaillent dans des environnements différents, utilisent des techniques distinctes et présentent, de surcroît, des modes de vie dissemblables. Les laguistas emmènent souvent toute leur famille lors des périodes de travail. Leur goût 
pour les boissons alcoolisées est plus prononcé : les dix «buveurs» (papudinhos) du petit village sont laguistas. Leurs enfants, dès les premières années, accompagnent le travail au lac et, quand ils sont au village, vont jouer librement à la rivière. Leurs habitations comportent d'ordinaire une seule pièce, presque sans meubles. Les pescadores, eux, divisent leurs maisons en plusieurs pièces, les meublent et les équipent à la façon urbaine; on peut même y trouver des lits au lieu des hamacs. Ils donnent aussi une éducation différente aux enfants, évitant qu'ils aillent jouer trop souvent à la rivière et ne les emmenant à la pêche que quand ils sont adolescents. Leur participation à l'organisation d'activités communautaires et leurs contacts avec les centres urbains sont plus soutenus.

Quant à la conformation physique des pêcheurs, leur musculature dorsale, de façon générale, est plus développée que l'antérieure, car dans les deux types de pêche, les mouvements qui nécessitent le plus de force et le plus de temps sont ceux qui rapprochent l'objet de l'homme, à savoir, le geste de tirer les cordes et les lignes (en mer) et la rame (dans le lac). Les lancers - de l'hameçon et du harpon- n'exigent ni beaucoup de temps ni une forte intensité, mais ils demandent de la précision et sont spécialement valorisés. On doit pourtant noter certaines différences. Les pescadores présentent une musculature spécialement développée au niveau des omoplates, en raison de la fréquence du geste de tirer vers leur thorax. Chez les laguistas, outre le renforcement notable de la région lombaire, les épaules sont rehaussées, avec les pointes des clavicules extrêmement marquées. Cela procède du geste de ramer, qui exige un appui et une rotation du torse pour tirer puis lever la rame. On dit aussi que les laguistas sont plus corpulents, parce que lorsqu'ils sont sur le lac, ils se reposent et se nourrissent mieux: "Ces gens qui travaillent sur le lac, tous sont forts, ils sont gros... Et ces pêcheurs en mer sont aussi comme ça? - Non, pas du tout, ce sont tous des gens maigres, ces types ne grossissent pas ». Autre observation concernant le corps des laguistas, de nature quelque peu taquine : ils seraient privés de juntas (d'articulations). En fait, la démarche des laguistas présente une raideur flagrante face à celle des pescadores, ce que certains attribuent aux longues périodes pendant lesquelles ils demeurent assis dans la pirogue, en une même posture, alors que le pescador doit à tout moment ajuster son corps au mouvement de l'embarcation.

ApprentissagesSur le lac

11 Dans les expéditions sur le lac (de 20 à 40 jours), on emporte des nourrissons, accompagnés par leur mère ou, s'ils sont en âge de se déplacer et de communiquer (vers trois ans), des enfants qu'accompagne leur père ou un autre homme. La première adaptation nécessaire pour l'enfant emmené à la pêche est de rester silencieux, ce qui est essentiel pour la pêche du pirarucu. L'adulte doit être suffisamment patient pour ne pas s'irriter outre mesure d'une attitude de l'enfant qui ferait fuir sa proie. Emmener un enfant (fils, neveu ou frère) de cet âge est quelque chose que les proeiros acceptent volontiers, bien que l'on assiste à certains rappels à l'ordre, avec de rares punitions (tapoter l'enfant de la hampe du harpon dans les cas extrêmes), ce qui arrive surtout lorsqu'on emmène deux enfants proches par l'âge. Pour que les petits puissent s'appliquer à l'art difficile de faire silence, on préfère en emmener un seul à la fois. Quand la pêche ne marche pas bien, le proeiro peut aussi laisser l'enfant dans la feitoria (habitation construite sur l'eau, que l'on utilise pendant les saisons de pêche), s'il se trouve quelqu'un pour les surveiller. On peut encore les laisser au village, ce qui est 
rare, surtout pour les pères, lesquels cèdent souvent à leurs fils désirant vivement les accompagner.

Il faut aussi savoir ne pas faire bouger la pirogue, bien souvent pendant plus de trois heures de suite. En fait, sur le lac, on ne fait jamais beaucoup de bruit -on parle en général plus bas, en peu de mots et les gestes sont silencieux-, et d'autant moins lorsqu'on entend un pirarucu qui vient respirer à la surface, car tout mouvement de la pirogue peut alerter le poisson et rendre sa capture impossible ou difficile. En se propageant dans l'eau, le moindre mouvement est perçu de fort loin par le poisson. C'est pourquoi l'immobilité nécessaire conduit jusqu'à supporter les piqûres d'insectes ou la soif avec impassibilité, ce à quoi l'enfant ne parvient qu'au bout d'un certain temps.

Pendant cette période d'apprentissage, l'enfant occupe le milieu de la pirogue, et il effectue des tâches secondaires et bien délimitées, comme prendre en main et ranger divers objets. Néanmoins, dès le début, le garçonnet porte une petite rame ainsi qu'une hampe proportionnée à sa taille, à laquelle s'ajoute une pointe en bois, puis en métal, selon son âge. Avec ce dispositif, il harponne de petits poissons ou des objets, à la condition que ce ne soit pas en des moments où le silence est indispensable. Les adultes l'aident à confectionner ces outils et réservent des occasions pour que l'enfant les emploie. Les petits peuvent harponner à volonté pendant de longues heures depuis la feitoria, et les adultes les entrainent également à partir de la pirogue. Quand il pleut, par exemple, des poissons d'une certaine espèce parcourent, désorientés, la surface de l'eau. À ces moments-là, les enfants s'amusent à harponner et les adultes les assistent. Dans ces situations s'exprime la profonde unité de l'apprentissage technique et de la formation du laguista en un sens plus large. Attentifs aux activités enfantines, les adultes font des commentaires et des plaisanteries qui renvoient tant aux habiletés techniques qu'aux aptitudes personnelles du néophyte.

Sont également très pratiqués deux types de jeux qui réunissent hommes et enfants dans l'eau pendant les moments de repos dans la feitoria. Au cours de l'un d'entre eux, un groupe doit toucher l'autre, qui tente de s'esquiver au fond pour parvenir à un piquet, chacun se trouvant "sauvé " dès qu'il l'atteint. L'autre jeu consiste à "harponner ", à partir de la feitoria, quelqu'un qui se déplace dans l'eau, avec une hampe légère dont l'extrémité n'est pas pointue. Dans ces deux jeux, les joueurs assument le rôle de différents animaux, suivant leur comportement dans l'eau. Les divers types de conduites des animaux aquatiques sont thématisés -leur vitesse, les profondeurs de leurs déplacements, leur agilité, leur production éventuelle de signes visibles à la surface, etc -, comme des considérations plus générales sur leur degré d'intelligence. Tout cela donne lieu à des commentaires et à des sobriquets qui deviennent courants dans la vie quotidienne au village. Le laguista le plus célèbre dans ces jeux est connu sous le nom de Ela (Elle), qui renvoie à la pirapema (Tarpon atlanticus), le plus agile de tous les poissons. Une certaine capacité pour la natation en surface, même si elle n'est pas très utile pour échapper aux « harponnages ", a valu à l'auteur de ces lignes la dénomination de Ariranha (Pteronura brasiliensis, un mammifère aquatique).

La genèse d'une habileté technique ou perceptive et le développement de propriétés personnelles ne peuvent pas être séparés dans les situations où l'enfant se trouve sur le lac. Quand plusieurs pescadores logent dans une même feitoria, formant ainsi un milieu masculin, les adultes jouent avec les garçonnets, presque toujours en soulignant des éléments de leur virilité et en les mettant en question. D'ordinaire, il s'agit de 
plaisanteries d'ordre sexuel, ou de remarques dubitatives concernant leur aptitude à accomplir quelque tâche relative à la pêche, ce qui est pris par l'enfant comme une offense. Ainsi, il arrive qu'un adulte engage une sorte de compétition avec les enfants pour pêcher des piranhas (Sarrasalmus rhombeus et Pygocentrus natterery) en dessous d'une feitoria ${ }^{3}$. Mais ce faisant, il s'arrange toujours pour que sa défaite soit possible; et il lance des défis et des provocations auxquels, espère-t-on, l'enfant répondra avec rapidité et véhémence, stimulé en cela par d'autres adultes. Restant à l'écart de ces jeux, le père occupe une position distincte: il procure l'alimentation et les soins élémentaires, en imposant des limites et en indiquant les devoirs de l'enfant dans cet environnement. $\mathrm{Si}$, pour l'enfant, cette situation revêt un caractère fortement agonistique, les adultes y voient un divertissement mineur, et il n'y a pas de tension entre le père et les adultes qui se livrent à ces jeux.

Toutefois, les adultes combinent pareils défis et amusements avec de chaleureux encouragements, s'adressant au petit comme s'il était un proeiro. Par exemple, quand une pirogue est en vue, au lieu que soit, comme d'habitude, par son proeiro qu'on l'identifie, on peut la saluer par le nom de l'enfant qui se tient au milieu de l'embarcation. Lorsqu'une pirogue arrive à la feitoria en apportant un pirarucu, c'est l'enfant qui peut être acclamé à la place de son père, de son oncle ou de son frère. De sorte que la conduite des adultes envers les enfants s'établit entre le défi et la reconnaissance.

On voit des enfants qui font déjà office de piloto à environ huit ans, mais l'âge des débuts varie suivant les familles et l'engagement du petit dans les activités scolaires. Il faut dire que tout homme désire vivement que les capacités à piloter de ses fils se développent rapidement, afin que, ces dernières étant acquises, le produit de la pêche ne soit pas partagé avec l'unité domestique d'un piloto « étranger ».

Le rapport entre le proeiro et le piloto se conforme à un code de conduites et de communications : celui qui se tient à la poupe doit apporter vitesse et bonne direction à l'embarcation; il doit aussi aider à repérer le poisson, surtout en arrière de la pirogue. Pourtant, quand il engage un piloto encore novice, le proeiro emploie ses compétences à mesure qu'elles se développent. Ainsi, dans le cas d'un enfant, on sait qu'il ne va que "maintenir la poupe " à l'instant du harponnage, sans avoir la force de suivre le poisson en fuite; par conséquent, le proeiro attend la meilleure occasion pour prendre le poisson au premier harponnage. Pour la même raison, le proeiro passe à la poupe pendant le trajet vers le lieu de pêche ou de retour au village, car l'enfant ne connaît pas encore le véritable labyrinthe qui traverse la mangrove et sa force ne suffit pas à virer serré, ni son endurance à ramer continuellement (jusqu'à vingt heures de suite).

Pendant cette période, le proeiro apprend au piloto à assurer ce qu'il attend de lui : la connaissance des trajets, comment positionner la pirogue pour le harponnage, etc.

Une fois acquise la maîtrise des tâches qui lui incombent, le piloto observe attentivement les stratégies du proeiro pour localiser et capturer le pirarucu. À ce stade, le piloto connaît depuis longtemps les types d'indices et de signaux grâce auxquels on repère le poisson. L'eau des lacs est trouble; à sa surface, trois indications permettent au laguista d'estimer où se trouve l'animal. La siriringa, ensemble de petites bulles qui émergent de la terre quand le poisson se déplace sur le fond; le carculo, ondulation ténue émise quand le poisson se déplace entre deux eaux et à une certaine vitesse; et le buio, qui dénote l'action du poisson arrivant à la surface pour respirer -la meilleure occasion pour le harponner. Par ailleurs, le piloto connait déjà les différents types de 
harponnage (photo 4). Dès lors, cette interaction avec le proeiro lui fournit la compréhension des stratégies d'approche et de capture dans chaque situation. Les explications orales existent, mais sont subsidiaires de l'observation des actions : on dit que les pilotos « doivent regarder pour savoir faire ».

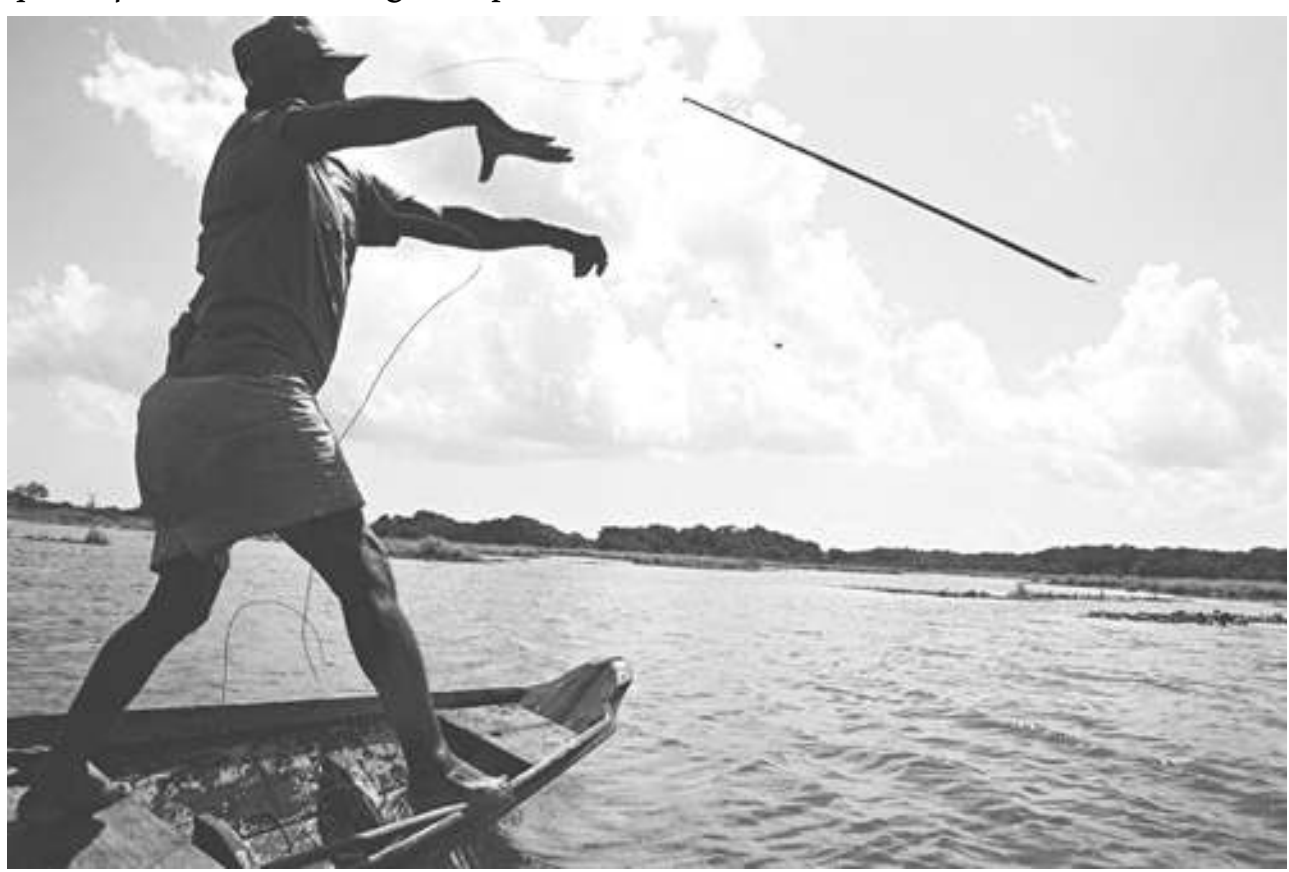

Photo 4. Borcagio lance le harpon. Les proeiros peuvent le faire avec précision jusqu'à plus de vingt mètres

21 Cependant, il faut encore " acquérir la pratique » (ganhar a prática). Le dimanche est le jour consacré à cette " acquisition », quand pilotos et proeiros échangent leurs rôles. Ce passage à la proue procure au piloto d'autres savoir-faire : harponner debout depuis la pirogue en mouvement, prendre des décisions et diriger l'activité. Il faut noter qu'alors, à la poupe se trouve quelqu'un de plus important que le piloto, quelqu'un qui peut le critiquer et le conseiller en toute légitimité. Si bien que, au moment de "prendre sa propre pirogue », le piloto est capable d'avoir pleinement confiance en soi («il se fie à son harpon »), car il a déjà tué un pirarucu.

Lâge auquel chaque laguista devient proeiro est très variable - de 14 ans à plus de 20 ans. En effet, même s'il est déjà techniquement préparé à le faire, son passage à la proue est entraîné par l'accession à un nouveau statut dans l'unité domestique de production : il "passe à la proue » lorsqu'il se marie, créant ainsi un nouveau groupe familial, ou si, dans sa famille d'origine, un adulte meurt, cesse de travailler ou abandonne le foyer. Ce passage dépend aussi de la sanction collective quant à sa compétence, qui s'exprime dans la concession du crédit (aviamento) grâce auquel le nouveau proeiro obtient la farine, le sel et d'autres produits nécessaires à sa première expédition. Quand les proeiros racontent leur vie, ils indiquent qui leur ont appris le travail et le patron (patrão) qui a reconnu leur aptitude pour la première fois.

Mais une fois le laguista devenu proeiro, de nouveaux apprentissages l'attendent. Les changements de hampe (en poids et en forme), les substitutions de piloto (vitesse et positionnement inédits), exigent de sa part une adaptation gestuelle. C'est aussi le cas des changements de lieux de pêche. Les proeiros préfèrent passer de longues périodes au même endroit, là où s'appliquent de la meilleure manière leurs aptitudes personnelles 
et où les caractéristiques de leur harpon s'ajustent le mieux à celles du milieu : mangrove vs lac, profondeur, déplacements des poissons, types d'indices observables... C'est pourquoi les lieux de pêche portent souvent le nom du laguista qui les fréquente. Mais il arrive que l'on change de lieu, soit parce que le proeiro l'a soudain décidé, soit que les adaptations en vigueur jusque là aient été rendues inefficaces par certains facteurs -oscillations saisonnières du niveau, propagation de l'eau de mer dans les lacs...

Passé un certain âge, entre 50 et 60 ans, la diminution de la force employée au lancer du harpon et (ou) la baisse d'acuité visuelle amènent le proeiro à «revenir à la poupe ». Cette situation nouvelle provoque parfois des conflits sur le statut de certaines des tâches assignées au piloto (cuisiner, laver l'embarcation...); mais elle s'avère problématique surtout parce que l'ancien proeiro éprouve des difficultés à positionner la pirogue selon les directives de l'autre pêcheur. Il a passé plus de trois décennies à appréhender l'environnement selon ses propres compétences, suivant sa manière de harponner, alors que maintenant il doit le percevoir en s'accommodant de celles de l'homme qui l'a remplacé à la proue.

Sur ce rapport entre la perception de l'environnement et les compétences acquises par les pêcheurs, voici une anecdote exemplaire. Il y a plus d'une décennie, l'eau salée a pénétré abondamment une partie des lacs, trois années de suite. La troisième fois, les pirarucus ne sont pas morts, et plusieurs sont même restés dans la zone salée. Comme ce phénomène a également entraîné une certaine transparence de l'eau, quelques laguistas se sont rendus dans cette région et se sont juchés sur un arbre, au-dessus d'un chemin, en espérant une capture aussi inhabituelle que prometteuse. Les poissons sont arrivés et les pêcheurs, qui, pour une fois, les voyaient nettement, les ont harponnés verticalement (c'est apparemment plus facile); or, ils n'ont pas pu les atteindre. Capables de faire des prédictions complexes sur la position du poisson sous la surface, mais pas de corriger la réfraction de son image dans l'eau limpide, ils se sont toujours trompés, fort étonnés les rares fois où cela s'est répété. Et aujourd'hui encore, on ne peut qu'attendre une nouvelle occasion de percer l'énigme : comment harponner un pirarucu bien visible?

En mer

Venons-en à la pêche en mer. Avant de commencer à pêcher en bateau, à environ 13 ou 14 ans, l'enfant ne connaît la vie à bord que s'il a dû faire un voyage en mer -situation bien différente de celle de la pêche. Par ailleurs, il existe d'autres activités antérieures à son initiation au travail qui méritent attention. Il sait effectuer plusieurs des opérations élémentaires : faire quelques nœuds, manier un outil important comme le couteau... Il a déjà acquis quelque connaissance des propriétés mécaniques de l'hameçon dans les pêches enfantines au bord de la rivière, ou à la plage avec un adulte. Pour ces raisons, et parce qu'il est présent lors du retour de pêche des adultes, le traitement du poisson ne lui est plus étranger. Au surplus, il est pénétré d'une cosmologie dont le mouvement des eaux constitue une manifestation centrale : cette temporalité particulière n'est nullement inédite aux yeux du garçonnet, du fait tant de l'influence décisive de la marée, qui scande la vie quotidienne du village, que pour le rythme des activités de pêche, dont l'apprentissage lui est possible même à terre.

Mais c'est une véritable fascination pour le bateau, très intense, qui caractérise un fils de pêcheur en mer; elle prend d'ailleurs beaucoup de son temps. Il dessine des bateaux partout, sur les cahiers scolaires, sur le sol et ailleurs. Il en fabrique des miniatures sous 
forme de maquettes, quelquefois équipées de moteurs à pile pour disputer des régates. Plusieurs matériaux sont employés pour confectionner ces petits jouets, comme des restes des fruits, du bois et du métal; de plus, quantité d'objets sont transformés pour faire office de bateaux -une cuvette à linge, un tronc d'arbre... ou même une antenne parabolique! Une description exhaustive de cette thématique dans l'imaginaire et les jeux enfantins exigerait plus d'espace, mais il faut ici mentionner l'intérêt considérable pour les « vrais » bateaux. Les embarca-tions amarrées près du village sont assidûment occupées par les enfants qu'il faut éloigner quelque peu, et non sans efforts, lorsque les pêcheurs doivent s'activer. La présence des jeunes est à ce point constante, leur curiosité tellement grande, que l'une des tâches des pêcheurs après le retour au village est de ranger soigneusement le bateau afin que les enfants ne puissent y voir les instruments susceptibles d'être endommagés ou d'en être retirés.

Quand le bateau s'en va à la pêche ou en revient, les enfants sont d'ordinaire à bord, car ils y montent ou en sautent dès que l'embarcation emprunte la rivière. Les retours de pêche comme les départs en mer entraînent presque toujours une petite pause dans les activités au village: les gens regardent les bateaux et échangent des commentaires parfois banals, mais souvent avec un certain lyrisme. Cependant, les enfants ressentent ce passage entre terre et mer avec exaltation, et ceux qui n'accompagnent pas les bateaux n'ont de cesse de bavarder et d'imiter le bruit des moteurs.

29 L'important est que tout cela ne paraît pas constituer une préparation lente et progressive de l'enfant à ce qu'il va découvrir au moment de son initiation dans un bateau de pêche. Autrement dit, et sans qu'on puisse nier que cette forme d'expérience concernant la pêche donne au jeune garçon une certaine "empreinte technique ", sa valeur semble consister à produire chez lui un "goût pour la pêche ", ce qui va être un facteur décisif de son adaptation et de l'ajustement durable de sa présence à bord.

La famille décide quand le garçonnet doit être initié à la pêche en mer. Sont pris en compte, pour ce faire, son développement physique et mental, ainsi que ses activités scolaires, outre le rapport consommateurs/ producteurs au sein de l'unité domestique. Lors de son premier voyage, il n'est pas forcément accompagné d'un proche parent, car ce qui détermine la composition des équipages est un système supra-familial. D'abord, le nouveau marin oriente sa volonté dans un processus nommé "s'habituer" (se acostumar); il sait d'avance que le manque de confort, les désagréments et la douleur peuvent atteindre un degré élevé, puis diminuer-mais il sait aussi que certaines personnes n'ont pas réussi s'y accoutumer. Aussi n'est-ce pas sans une forte inquiétude que, les premières fois, le jeune garçon monte à bord, déjà responsable du maintien de sa "disposition », et tenu de montrer qu'il est "actif au service» dans une situation pénible. Comme le dit un pêcheur à propos de l'initiation de ses fils : «Ils devaient encaisser les coups durs. Ici, ils mettaient l'appât à l'hameçon en vomissant, pendant qu'un autre vomissait par là, mais il leur fallait bien faire le boulot ».

31 Le garçon est surtout soucieux de tenir bon, de garder un esprit résolu, car "s'habituer » et apprendre les tâches sont des choses qui dépendent du temps et de sa présence active à bord. Il gagne en compétence justement parce qu'il est à bord et qu'il y réalise les tâches qui lui sont assignées. Il ne s'agit pas ici d'imputer aux pêcheurs un manque de désir d'éduquer les néophytes. En fait, le débutant trouve toujours des pêcheurs plus attentionnés que d'autres et qui s'aperçoivent qu' «il est actif ». Ceux-ci ont à cœur de lui apprendre à faire quelque nœud où à couper le poisson d'une certaine façon, durant les moments où c'est possible. Mais pendant l'activité de pêche, la 
succession des opérations ne peut pas être interrompue. Dans l'ensemble des tâches, sont affectées au débutant les moins centrales et les et moins dangereuses; reste qu'elles font partie, comme les autres, d'un système qui fonctionne de façon coordonnée et à une allure déterminée. Quand le novice ne sait accomplir quelque besogne, le pêcheur expérimenté ne peut que lui signaler son erreur ou le cas échéant, accourir et prendre les choses en mains avant de revenir à son poste. Cela parce que, s'ajoutant aux pêcheurs, s'inscrivent dans ce système des éléments dont statuts et modalités d'action sont fort divers, et dont les effets ne sont guère perturbés par la présence d'un débutant. La marée, l'hameçon, la ligne, le moteur l'embarcation ellemême: toutes choses avec lesquelles il doit interagir en s'arrangeant de leurs caractéristiques.

Il ne s'agit pas d'une modalité orientée d'apprentissage. Il serait plus juste de parler d'une immersion dans le travail, par suite de l'effort du débutant pour s'ajuster au mieux à la vie à bord, dont le rythme reste, bien sûr, indifférent au déphasage qu'il provoque chez le néophyte. L'apprenti devient un pêcheur de plus au travail en étant actif, en ayant toute disponibilité pour le faire. Interrogé sur son apprentissage, le pescador ne peut que faire référence à son insertion dans cette dimension autoorganisée du travail à bord:

Eh bien j'ai travaillé comme ça, «en camarade ». Je me mettais comme camarade dans un bateau. Après, là-dedans, j'ai pratiqué -lancer d'hameçon, entretien du poisson, tout, quoi. Et ça a été bon, j'ai tout appris. Je savais lancer l'hameçon, décapiter le poisson et l'ouvrir, couper, saler, tout.

33 Comme le rythme des opérations, la maîtrise de l'horaire (horário) est acquise à bord. Là, le débutant apprend comment la marée gouverne la temporalité sur le bateau à plusieurs niveaux. La sortie pour la pêche attend toujours «l'eau tirer » (a água puxar), c'est-à-dire les marées des jours de lunes pleine et nouvelle. La présence en mer peut ne durer que le temps d'une marée descendante (environ huit heures) ou aller jusqu'à "deux lunes» (21 jours); cela dépend de la saison, du lieu de pêche, etc. Lors du déroulement de la pêche, les phases d'activité -comme lancer et retirer la ligne- sont déterminées par les changements de direction et d'intensité du flux aquatique. Mais la marée oriente aussi un troisième niveau de cette temporalité : celui de l'articulation entre la gestuelle du pêcheur, le bateau et l'outillage. Pour marcher sur le pont quand la mer est agitée, le pêcheur doit tenir compte de l'accélération que le mouvement de l'embarcation imprime à son propre corps et en prévoyant le balancement du pont qui surviendra à la lame suivante. Pour boire de l'eau, on doit incliner son verre en considérant la force de déplacement que la vague va donner au liquide.

On peut affirmer que pour une bonne part, l'habituation du pêcheur n'est autre que l'ajustement à cette temporalité propre au bateau. Travailler, se nourrir et se reposer en des moments différents de la journée à chaque marée, sont des incommodités qui marquent la mémoire. Mais les seuls faits de ne pas vomir, de ne pas tomber et d'avoir les mains libres pour le travail sont fonction d'une bonne adaptation au roulis et au tangage du bateau - «le pescador doit être sûr face aux vagues». Et la profondeur organique de cette adaptation est démontrée par les périodes d'insomnie que subissent, une fois revenus à terre, plusieurs pescadores, touchés par les troubles cinesthésiques qui résultent des vagues. À ce propos, on aime mentionner un ancien pêcheur qui ne pouvait dormir à terre que si quelqu'un le berçait dans un hamac au rythme de la mer. Il faut comprendre que la période requise pour s'habituer à la vie en mer ne peut pas être conçue tout simplement comme un temps d'acquisition d'adresses particulières. 
C'est plutôt une transformation, qui implique aussi la perte de différentes aptitudes pour la vie à terre:

Personne ne peut rien contre la mer. Même un grand navire, la mer le fait bouger. La vie en mer est faite de sacrifices, c'est une vie misérable pour ceux qui ont de la peine à s'habituer. Mais il y a des gens qui s'embarquent et qui s'habituent tout de suite! Et il y a en aussi qui s'habituent plutôt en mer qu'à la maison, à terre. Tu le savais?

Au sujet de l'importance de la marée sur cette transformation adaptant l'individu au travail à bord, on doit noter qu'à l'expression « la marée court », peuvent être ajoutés les adjectifs « vite » et «fort ». C'est-à-dire qu'elle est à la fois évolution dans le temp et force; elle a vitesse et poids. Et c'est justement à la force du courant que le bateau, le moteur et les engins sont liés. Ainsi, de même que l'aspect temporel de la marée, son aspect moteur débouche sur des ajustements du pêcheur; et là, ils sont plutôt d'ordre morpho-fonctionnel : «Les premières fois qu'on tire [la ligne], on attrape des cals aux mains, parce que la marée court... »

Or, ces adaptations à la force de la marée sont contraires à la vie à terre, constatent les premiers intéressés: si le pescador a besoin de mains endurcies pour le travail en bateau, cela le prive de dextérité pour des manipulations plus raffinées, pour caresser une femme ou un enfant, pour tenir un crayon...

Sans entrer dans les détails du travail à bord, disons que les pescadores ne sont pas spécialisés. Certes, un des pêcheurs est le "chargé » (encarregado) du bateau, qui coordonne les activités, sale le poisson et, le plus longtemps, contrôle le moteur et le gouvernail. Actuellement, le chargé d'un bateau peut être un jeune pescador, avec un ou deux ans d'expérience. Il obtient cette position car le bateau appartient à sa famille ou parce qu'il a la «confiance » de son propriétaire. Des modifications de la pêche côtière au Sucuriju, dues aux changements économiques des trois dernières décennies, ont fait qu'aujourd'hui, la compétence à gérer le travail à bord et la « responsabilité » sont les principales caractéristiques demandées à un chargé : au cours de cette période, la part relative de sa connaissance des techniques et de la mer s'est réduite. Outre la manutention de la technique de capture, d'après les pescadores, les autres facteurs sont devenus plus faciles à contrôler : la pêche s'est rapprochée de la côte, d'où l'on peut toujours avoir un contact visuel avec la terre, les bateaux sont moins grands et équipés de moteurs. Alors, " au Sucuriju on ne fait plus de chargés comme jadis », parce qu'on ne fait plus de pêche comme jadis : le système technique a changé et, avec lui, le rôle même des compétences humaines sur le bateau.

D'autres activités sont effectuées par n'importe quel pêcheur, sauf le lancer de l'hameçon. Cette tâche n'est ni un apanage ni différemment rémunérée, et tout pêcheur n'est pas forcément désireux d'en être l'agent, mais c'est la plus prestigieuse. Je la décris brièvement: pour positionner la ligne au fond, le bateau se déplace tout droit dans une direction telle que le flux de la marée progresse du côté droit de la proue au côté gauche de la poupe; la palangre est progressivement lancée en mer, avec les trois types d'objets qui la composent : les ancres, les bouées et les hameçons, tous attachés à la ligne. Ensuite, étant donné que le bateau se déplace, la ligne qui est à bord va en sortir à un certain moment et le rôle du pêcheur est de lancer les hameçons à l'eau avant qu'ils n'y soient entraînés par la tension de cette ligne. Il faut les lancer un à un, dans un ordre défini et à un rythme moyen de moins d'une seconde par hameçon. Entre chaque groupe de quarante hameçons, il y a une pause de quelques secondes, quand le lanceur attend que soient lancées une ancre et sa bouée par d'autres pêcheurs. Tout 
cela prend environ dix minutes et doit conférer une tension optimale à la ligne. Si le pescador lance plus vite, la ligne reste lâche ou enroulée; s'il lance plus lentement, l'hameçon peut «sauter » de ses mains, avec la possibilité de l'accrocher et de l'amener au fond. Pour parer à une telle éventualité, le lanceur d'hameçon porte à la ceinture un couteau avec lequel il pourrait couper la ligne pour revenir en surface (photo 5).

En fait, toutes les fonctions doivent être bien coordonnées, mais le lancer de l'hameçon est l'expression même de la maîtrise du rythme, car là, le danger et donc l'agilité nécessaire sont plus évidents. C'est la raison du surplus de prestige qu'il confère. De plus, son accomplissement en bonne et due forme dépend des dispositions du pêcheur. Il y a des jeunes qui lancent l'hameçon dès leurs premières sorties en mer et des pêcheurs âgés qui n'ont jamais eu le courage de le faire.

Il y a des gens qui deviennent plutôt nerveux quand ils vont lancer l'hameçon. Moi, la premières fois que j'suis allé lancer, j'étais tellement nerveux que mes jambes tremblaient, comme ça. J'avais déjà beaucoup travaillé à la pêche, mais j'avais jamais lancé l'hameçon. Je disais «Un de ces jours, je vais essayer ». Une fois, on était à la pêche, la marée courait beaucoup, alors mon frère m'a dit « Tiens! C'est l'moment d'essayer! » J'lui ai demandé «Tu crois? » Et lui : « Vas-y, qu'il va rien t'arriver ! Après qu't'auras lancé une première fois, tu voudras recommencer tout le temps. » J'ai dit « Ça va! » Alors j'suis allé lancer. J’ai bien lancé mais j'étais très nerveux et ça m'a un peu embêté. On imagine toujours ce qui peut arriver, que les choses peuvent ne pas bien se passer. Après qu'on a lancé une fois, on a toujours envie d'lancer.

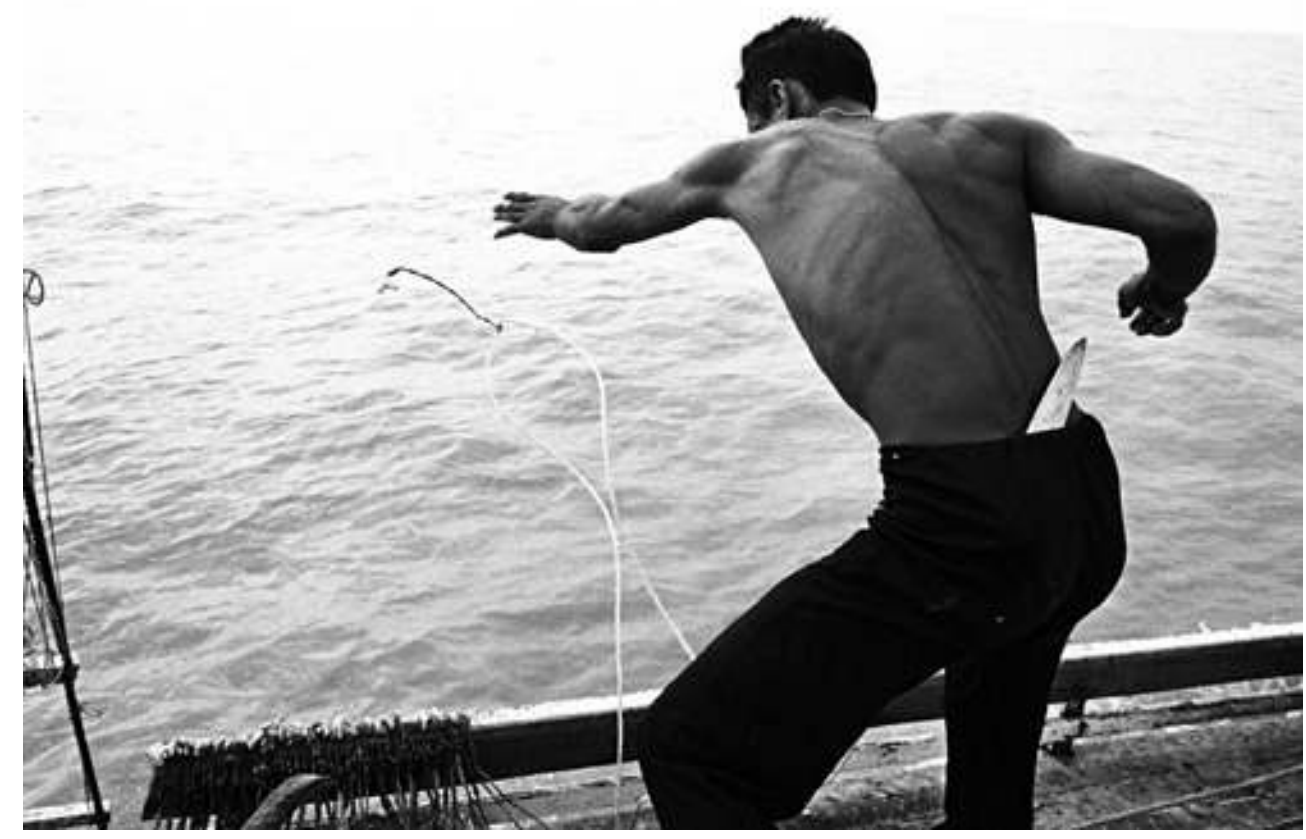

Photo 5. Gero lance l'hameçon. À sa ceinture, le couteau.

Au-dessous et à gauche, on peut voir les autres hameçons qui seront lancés ensuite

Mais de nouvelles adaptations sont toujours à l'horizon du pêcheur, car comme partie d'un ensemble, il doit se faire à tous les changements. On cite le cas d'un pêcheur qui a refusé pendant un an l'enviable proposition d'être chargé d'un nouveau bateau, car « il tombait beaucoup dans les vagues ». Néanmoins, après avoir fait quelques voyages comme marin, il put «s'habituer» et accepter enfin la proposition. En revanche, les modifications de l'organisme du pêcheur lui-même exigent de nouvelles stratégies. Souffrant de l'estomac, un pêcheur expérimenté a commencé à prendre des 
médicaments contre le mal de mer pour conserver son activité à bord. Autre cas, un vieux pêcheur, régulièrement gagné par une somnolence irrésistible, a trouvé une solution sui generis pour éviter de tomber à la mer -risque que courent normalement les débutants : à 79 ans, il conduit encore de longs trajets nocturnes, mais à présent attaché au bateau...

Passages

41 Je viens de décrire les particularités de deux types de pêche bien distincts. Mais ces domaines d'activité ne sont pas séparés par une frontière étanche. En effet, l'arrivée d'un pescador sur le lac ou la présence d'un laguista à la pêche en mer ne sont nullement prohibées : elles s'avèrent même courantes. Pourtant, suivant leurs sens, les passages de l'un à l'autre milieu traduisent des stratégies d'adaptation différentes. Pour deux raisons, la transition du lac vers la mer est plus courante, qu'elle soit temporaire ou définitive. D'abord parce que, normalement, un marin pêcheur gagne plus qu'un piloto. Une saison en mer est par conséquent une bonne solution quand l'accès au lac est difficile, comme en saison sèche. Mais la transition définitive est le fait de ceux qui pensent ne pouvoir jamais devenir proeiro ou qui ne s'affirment pas dans cette position. La supériorité des gains en mer entraine la rareté des pilotos avec femme et enfants. On trouve en général dans la position de piloto un ancien proeiro, un célibataire, un jeune de la famille du proeiro ou l'épouse de celui-ci. Seconde raison : le changement en faveur de la pêche en mer survient quand une unité domestique qui exploitait le lac devient propriétaire d'une embarcation maritime (cas fréquent au cours de la dernière décennie, du fait d'un programme de financement de l'État). Quoi qu'il en soit, temporaire ou définitive, le passage du lac à la mer exige du laguista une disposition et une grande volonté de travail comme de (ré)adaptation. Malgré leur insistance explicite sur les possibilités de pêcher aussi en mer, la majorité des laguistas ne pense certes pas à subir la dure adaptation qui serait alors nécessaire.

Dans le sens inverse - de la mer vers le lac-, on change moins souvent. De façon temporaire, la transition se produit lorsque le pescador n'a pas la possibilité de sortir en mer, soit à cause des orages de février-mars, soit en raison d'une avarie au moteur, d'un dommage de l'embarcation ou des engins de pêche, ou encore s'il manque d'autres éléments au bateau (marins, carburant, sel...). Les pescadores trouvent agréable et confortable le séjour sur le lac, mais ils craignent les longs trajets à rames pour aller à la zone de pêche et en revenir, outre les animaux et les autres êtres qui la peuplent. On observe que lorsque le pescador va sur le lac, il se livre à des types de pêche différents de ceux que pratiquent les laguistas, ou alors il fait fonction de piloto-jamais il n'est proeiro.

43 Si donc il existe certaine perméabilité entre les deux domaines, ceux-ci apparaissent très bien marqués. Où qu'ils se trouvent, les pêcheurs sont identifiés ou bien comme laguistas ou bien comme pescadores, et la transition définitive entraîne un changement d'identité. Ce dernier est publiquement attesté quand un pêcheur change de groupe à cause des jeux et des compétitions qui opposent les laguistas aux pescadores à chaque festivité, surtout dans lors du plus important événement rituel du village, la Festa de Nossa Senhora de Nazarét.

Laguistas et pescadores

On a vu que l'apprentissage de la pêche sur les lacs consiste à développer un savoir quant au comportement du poisson, et des rapports très sophistiqués avec le milieu en matière de percepto-motricité, lesquels demandent plusieurs années d'expérience et 
d'«investissement» (dedicação). Le proeiro lui-même est l'épicentre du système technique et, comme on l'a vu, la dynamique du travail au lac est toujours ajustée à sa formation; d'ailleurs, il existe des institutions qui assurent son développement, comme le passage dominical de la poupe à la proue. En mer, en revanche, sont au centre de la pêche des forces (marées et moteur) et des instruments (ligne, hameçons et ancres) rangés selon un ensemble mécanique qui détermine des rythmes de travail assez stricts. En plus de ces contraintes, le rôle même du pescador dans pareil système s'établit en grande partie sur des compétences dont l'acquisition est plus ardue qu'élargie dans le temps. Bien entendu, l'habileté a sa valeur, et on ne fait pas tout d'un coup un lanceur d'hameçon adroit; cela dit, un débutant capable d'« être actif » à bord est volontiers accepté dans un équipage, à la différence du pescador expérimenté mais qui ne pourrait pas suivre le rythme du travail. C'est pourquoi l'engagement dans la pêche maritime dépend moins du temps d'apprentissage des gestes techniques que du succès de la fusion intense du jeune homme dans le travail sur le bateau. Enfin, pour souligner la différence des apprentissages en chaque cas, on peut proposer cette comparaison: le proeiro passe plus de six ans à tester de diverses façons l'élément principal de son métier-le harponnage; en revanche, le pescador ne prend connaissance du rythme des tâches sur le bateau que lorsqu'il va à bord pour la première fois. Peut-être suffirait-il de dire qu'il y a plusieurs jeunes gens qui sont restés pendant des années à travailler ou à étudier dans des villes, ou quelques étrangers au village qui y sont venus pour s'employer à la pêche en mer -à la différence de tous les proeiros qui, eux, sont nés au village et élevés dans une famille de laguistas ${ }^{5}$.

Mais il faut en dire un peu plus. Outre l'apprentissage d'un métier, l'accès aux fonctions de pescador ou de laguista signifie "être macho" grâce au travail. Mais la masculinité semble distincte en chaque cas. Sur le lac, elle s'affirme par la capacité de capturer le poisson lors d'un duel dont les connotations sexuelles sont multiples ${ }^{6}$ et les effets profonds sur le statut personnel de l'homme. En mer, le pescador se forge moyennant sa motivation à supporter les conditions fort difficiles du travail à bord. Dès lors, la reconnaissance de son effort par les autres pescadores conditionne sa masculinité. Mais elle peut aussi être affirmée par les signes corporels de sa transformation : les jambes capables de le maintenir en équilibre, les mains rendues rugueuses par le sel et la tension des cordes, le visage ridé par le soleil et le vent.

Ainsi la formation de chacun de ces types de pêcheurs comporte-t-elle une épaisseur symbolique qu'il faut souligner. Commençons pour une constatation qui semble paradoxale : les laguistas affirment constamment que le harponnage n'est pas une chose difficile à apprendre, et ils disent même qu'il ne faut pas beaucoup de temps pour en maitriser la technique. Du reste, on dit que le fils d'un proeiro naît avec le « don » de harponner. Il ne va pas de soi, cependant, que tous les proeiros soient de bons harponneurs. En principe, "tous tuent» et "tous ratent", mais les différences existent. La question est que l'efficience n'est pas considérée comme le résultat en droit ni de l'habileté du proeiro ni de son application au travail -elle provient de facteurs extra-individuels de plusieurs ordres.

On sait, par exemple, que le pirarucu joue un rôle important dans sa propre capture. D'abord, tout harponnage n'est effectif que si le poisson « s'épouvante " (se espanta) au bon moment, pour permettre au harpon de "passer entre ses écailles». Mais ce n'est pas tout, car auparavant, le poisson doit se présenter de lui-même au laguista. 
Tu peux être bon pour le pirarucu, et alors il vient mourir à ton côté. Mais s'il s'est méfié de toi, il passe autre part, il ne te regarde pas. Il vient. Tu le rencontres et il vient, il vient, il vient. Et quand il arrive là il s'arrête. Tu peux harponner toute la journée, il n'est pas là.

La « défiance » (desconfessão) du poisson a son origine dans un événement passé, quand sa chair a été rejetée par quelqu'un ou si l'on a commis une faute dans le traitement de ses restes. Cela nous conduit à rappeler que le proeiro ne pense pas du tout à la capture comme à un problème d'adresse au harpon. C'est un a priori, que l'on peut généraliser à tous les proeiros, tout autant que la figure d'un proeiro sans harpon ou qui ne le maitrise pas bien représente l'absurde ou le ridicule. Les vrais problèmes se posent en termes de rapports de " confiance " avec le poisson, condition de cette capture qui est en fait un duel. D'autres exemples démontrent que le laguista est perméable aux types d'influence les plus divers. Son principal malaise physique -la rasgadura, sorte de blessure musculaire - ne peut être l'objet d'aucune attention prophylactique, car elle arrive sans motivation apparente : "Même sur le hamac on se rasga ". Et dans le lac, la folie même arrive soudain, provoquée par la rencontre fortuite et malchanceuse avec quelque animal ou être surnaturel.

En définitive, après avoir parcouru le long chemin pour "acquérir la pratique », le laguista "se fie à son harpon "; sans doute, mais il ne considère jamais qu'il gère son propre succès. Pour avancer une métaphore musicale, disons qu'en devant agir à l'égard d'êtres très divers, d'apparences et d'intentions qui ne sont presque jamais évidentes, le proeiro ressemble plutôt à un soliste qui se dédouble pour jouer avec un orchestre inconnu et privé de chef. Mais il ne peut qu'entendre ce solo toujours imprévisible comme l'expression de son identité propre.

Dans le système du bateau, les pescadores apparaissent quelquefois comme des moteurs au deuxième degré (quand ils tirent l'ancre ou la ligne, par exemple), mais ils sont surtout des mécanismes qui transmettent et orientent la force du bateau -laquelle est fournie par les effets de la marée et du moteur lui-même sur la tension de la ligne. Et justement, le pescador est pensé de cette façon. De même qu'un mécanisme, s'il est préparé pour bien fonctionner et le plus longtemps possible, tant mieux. Le pescador acquiert du prestige s'il est prêt à travailler à chaque marée, avec des intervalles réduits pour se reposer et se nourrir; s'il est capable de lancer l'hameçon le soir, parfois sous la seule lumière de la lanterne qu'il tient avec la bouche; s'il se propose pour tirer la ligne même s'il pleut; s'il n'a pas besoin d'être remplacé à la proue quand il tire la ligne, en étant capable de supporter la douleur aux mains, etc. De telle sorte que le pêcheur est considéré comme un composant du système, que ses propriétés sont équiva-lentes à celles des autres éléments du bateau, en termes de force, de résis-tance et d'efficience. Opèrent ici des analogies que l'on repère aussi bien sur le plan discursif - «L'eau salée ne peut venir à bout ni de l'acier inox [des embarcations] ni de la peau du pêcheur »-qu'au niveau pratique : l'huile lubrifiant les moteurs est utilisée pour guérir une douleur ou cicatriser une blessure, et pendant la pêche, s'effectuent à la fois le nettoyage du bateau et la toilette du pêcheur, avec de l'eau de la mer et la même brosse.

51 Le travail à bord est la voie de la "fabrication du pêcheur » au moyen du corps, mais aussi la cause de ses maux -folie, épuisement, rhumatismes. Et, si l'on se souvient que tout cela s'associe au thème de la volonté, on pourrait identifier dans l'apprentissage de la pêche en mer les trois éléments caractéristiques d'une pratique ascétique: disposition d'ordre moral, expérience corporelle « anormale et pénible ", processus de 
transformation. Mais ici, l'ordre s'inverse : le pêcheur doit partir d'un état d'esprit résolu et solide pour parvenir à la reconfiguration corporelle nécessaire au service à bord. S'en tenir à ce seul domaine, voilà qui définit ce procès: devenir pêcheur. $S$ 'habituer (se acostumar) ne signifie pas autre chose que « rester longtemps à travailler à la pêche ». Aussi le pescador accompli n'est-il pas considéré comme le fruit d'une action continue sur soi ni d'une transmission de connaissances, mais comme le résultat d'une immersion volontaire dans le système auto-organisé du bateau. Bref, c'est au pescador de présenter la «disposition " pour accéder à la pêche et y rester actif, mais une fois sur le bateau, c'est la dynamique des tâches et des conditions de vie à bord qui régit sa transformation.

\section{BIBLIOGRAPHIE}

Delbos, Geneviève \& Paul Jorion

1984. La Transmission des savoirs. Paris : Éditions de la Maison des sciences de l'homme.

Geistdoerfer, Aliette

1980. La Pêche côtière aux Iles de la Madeleine - Québec. Thèse pour le Doctorat d'État.

Université Paris V - René Descartes.

Ingold, Tim

2000. The Perception of the Environment : Essays on Livelihood, Dwelling and Skill. London :

Routledge.

Leroi-Gourhan, André

1984. O Gesto e a palavra I : Técnica e linguagem. Lisboa : Edições 70 (1ère édition 1964).

1987. O Gesto e a palavra II : A memória e os ritmos. Lisboa : Edições 70 (1ère édition 1965).

1992. Évolution et techniques I : L'homme et la matière. Paris : Albin Michel (1ère édition 1943).

\section{NOTES}

1. Je n'aborderai pas ici la question de savoir si les activités lacustres devraient être caractérisées comme pêche ou comme chasse. Limitons-nous à constater que parmi les indigènes eux-mêmes, les désignations varient, mais que la première prévaut.

2. Le pirarucu est un poisson que l'on trouve uniquement en Amérique du Sud, spécialement dans les eaux peu profondes, troubles et calmes au bassin de l'Amazone. Outre une respiration aquatique, il doit venir à la surface pour sa respiration aérienne. À Sucuriju, les plus grands spécimens pèsent jusqu'à $110 \mathrm{~kg}$ et mesurent jusqu'à 2,60 m. Un fois salée et séchée, on envoie la chair de ce poisson à Macapá et Belém, capitales régionales où elle est très appréciée. La gurijuba est un poisson estuarien et maritime qui se nourrit au fond des eaux peu profondes. Sa vessie natatoire (grude), qui a une grande valeur sur le marché international (tant pour la consommation humaine que pour la production de colle), est exportée via Belém. Sa chair n'est que salée, et 
commercialisée à bas prix dans l'État du Pará. Elle rapporte au pêcheur trois fois moins que la grude.

3. Les piranhas se nourrissent des restes de la production et des repas des laguistas; toujours sous la feitoria, ils constituent aussi une réserve assurée d'aliments pour les journées improductives. Cette symbiose carnivore s'accompagne de certaines « règles de commensalité » qui sont souvent transgressées de part ou d'autre.

4. Neuf nuits de célébration et de fête en remerciement à la patronne du village, Notre Dame de Nazareth, ont lieu pour avoir accompli une promesse originelle, qui demandait l'ouverture de l'embouchure de la rivière. Cette transformation (identifiable aussi sur des cartes anciennes), qui est à l'origine du village, a été réalisée par un énorme sucuriju qui a donné son nom à la rivière et au village. À l'occasion de cette festivité, laguistas et pescadores s'affrontent : quel est le groupe qui apportera l'offrande la plus importante à la Sainte ? Sorte de potlach catholique...

5. Il y a une seule exception, dont la biographie et le statut personnel spécial confirment la « règle».

6. Plus de dix expressions et gestes désignent aussi bien le harponnage que l'acte sexuel.

\section{RÉSUMÉS}

Les pêcheurs d'un petit village de la côte de l'Amazonie se sont partagé la spécialisation dans deux types de pêche pratiqués au sein de milieux différents, avec des systèmes techniques et symboliques spécifiques. Sur le lac, l'efficacité de la pêche dépend de la "pratique " du pêcheur lacustre (laguista), c'est-à-dire de l'adresse au harpon, de la profonde connaissance du comportement du poisson pirarucu (Arapaima gigas), comme d'un rapport intersubjectif avec certains animaux et des êtres surnaturels. En mer, s'est révélée centrale la «disposition » du pêcheur (pescador) pour une pénible adaptation corporelle au système de travail sur le bateau, où il est mis en rapport avec les instruments, le moteur et le flux de la marée. L'intégration des débutants évolue différemment dans chaque contexte -longue et graduelle pour le lac, soudaine et intense en mer. Dans ces deux processus, les apprentissages des habiletés techniques jouent des rôles distincts, en produisant les identités et les configurations personnelles spécifiques des laguistas et des pescadores.

The apprenticeship of fishing in a coastal region of Amazonia (Vila Sucuriju, Brazil) The fishermen in a small village on the coast of Amazonia share work specialization between two kinds of fishing carried out in different environments, each possessing specific technological and symbolic systems. On the lake efficient fishing calls for " practical experienc ", that is to say skill with a harpoon, and a thorough knowledge of the behavior of the pirarucu fish (arapaima gigas), as well as an intimate personal relationship with certain animals and supernatural beings. At sea, what has turned out as pivotal is the readiness of the fisherman to adapt to the very difficult physical conditions of work on the boat, where he is obliged to work with instruments and service the motor, all the while taking into account the ebb and flow of the tide. The integration of beginners follows a different rhythm in each context long and gradual for the lake, intense and rapid on the sea. In both of these apprenticeship processes, the way in which technical skills 
are acquired plays a distinct role in determining identities and specific social patterns of behavior.

Laguistas y pescadores. El aprendizaje de la pesca en una región costera de Amazonia (Vila Sucuriju, Brasil).

Los pescadores que viven en un pueblecito de la costa del río Amazonas se han repartido las especializaciones en dos tipos de pesca, practicados con sistemas técnicos y simbólicos específicos en el marco de medios naturales distintos. En el lago, la eficacia de la pesca depende de la «práctica » del laguista, es decir de la habilidad con el arpón y del conocimiento profundo del comportamiento de un pez: el pirarucu (Arapaima gigas), así como de relaciones intersubjetivas con ciertos animales y con unos seres sobrenaturales. En el mar, lo que se reveló central fue la « disposición » del pescador respecto a una adaptación corporal penosa al sistema de trabajo en el barco, donde tiene que ser relacionado con los útiles, el motor y el flujo de la marea. Los aprendizajes masculinos evolucionan de manera particular en cada contexto - son largos y progresivos en el lago, repentinos e intensos en el mar-, produciendo las identidades y la configuraciones personales propias de los laguistas y de los pescadores.

\section{INDEX}

Mots-clés : apprentissage, amazonie, Brésil, techniques de pêche, pêche côtière, pêche lacustre Keywords : apprenticeship, amazonia, Brazil, fishing technology, coastal fishing, lake fishing

\section{AUTEUR}

\section{CARLOS EMANUEL SAUTCHUK}

Doctorant en anthropologie sociale à l'Université de Brasília, sujet de recherche : Le harpon et l'hameçon : technique et personne dans une région côtière de l'Amazonie. Chercheur invité à l'équipe «Techniques et culture » (CNRS/MNHN) pendant l'année 2005/2006, avec le soutien du Ministério da Educação do Brasil - CAPES/MEC. Bénéficiaire d'une bourse brésilienne du Conselho nacional de desenvolvimento científico e tecnológico-CNPq. Cet article présente des données et analyses initiales d'une recherche doctorale; je remercie Jean-Luc Jamard pour son aide à la traduction du portugais (Brésil). 